\title{
Original
}

\section{Características clínicas y sociolaborales de pacientes psiquiátricos al término de la incapacidad temporal por contingencia común}

\author{
Clinical, social and labour features of psychiatric patients at the end \\ of temporary nonoccupational disability
}

\author{
Pastrana-Jiménez $\mathrm{Jl}$ ', Fernández-Labandera $C^{1}$, Ramos-Muñoz R ${ }^{1}$, Carrasco-Perera $\mathrm{JL}^{2-3}$ \\ 1. Dirección Médica de Contingencias Comunes. Servicios Centrales de Ibermutuamur - Corporación Mutua. \\ Madrid. \\ 2. Servicio de Psiquiatría Hospital Clínico Universitario San Carlos. Madrid. \\ 3. Departamento de Psiquiatría de la Universidad Complutense. Madrid.
}

Recibido: 20-02-13

Aceptado: 21-05-13

\section{Correspondencia}

José Ignacio Pastrana Jiménez

C/ Ramírez de Arellano 27

28043 - Madrid

Tfno: 917445100

joseignaciopastrana@ibermutuamur.es

Resumen

Introducción: La patología psiquiátrica es la principal causa de pérdida de calidad de vida y presenta unos altísimos costes, personales y económicos. Pese a ello, existen pocos datos sobre las características de los pacientes psiquiátricos con incapacidad laboral y sus características clínicas. Por ello nos proponemos describir estas características en los pacientes con más de 12 meses de incapacidad temporal e investigar factores que puedan estar ligados a cronicidad o disfunción.

Material y métodos: Sobre el total de pacientes derivados por el INSS para evaluación psiquiátrica, se excluyeron aquellos que no se encontraran en Incapacidad Temporal o que llevaran menos de 12 meses, conformando una muestra de 97 pacientes. Se registraron las principales características sociolaborales y clínicas, diagnóstico según criterios DSM-IV-TR, se aplicaron escalas de funcionalidad e intensidad sintomatológica como EEAG, MADRS y WHODAS O.

Resultados: La distribución por sexos fue homogénea, con una edad media de 47 años, vive en pareja el $51,5 \%$, normalmente refieren una vivencia de apoyo social moderado y $1 / 3$ no ha superado estudios primarios. El 78,4\% presenta antecedentes físicos importantes, fuma 50,5\%, 59\% tiene antecedentes familiares psiquiátricos, que tienden a asociarse a baja funcionalidad y tratamientos más prolongados. La concordancia diagnóstica es del $71,1 \%$, los trastornos afectivos son el grupo diagnóstico más frecuente. El trastorno adaptativo es el diagnóstico más frecuente en el grupo al que no se le encuentra limitación funcional y se asocia a menos tiempo en tratamiento y a menos intensidad sintomatológica. Las escalas utilizadas correlacionan entre sí, distinguiendo EEAG y WHODAS O a los trastornos adaptativos y a los cuadros no incapacitados, mientras que MADRS señala a los trastornos afectivos. Tras la evaluación, al 46,39\% no se le propone incapacidad, al 11,34\% se le solicita prórroga, al 29,90\% incapacidad permanente para su trabajo, y al $12,37 \%$ incapacidad permanente para cualquier trabajo. Los pacientes a los que se les recomendó incapacidad permanente presentaban asociación a bajo apoyo social, tiempo prolongado en seguimiento psiquiátrico, antecedentes familiares psiquiátricos y diagnóstico distinto a trastorno adaptativo. 
Conclusiones: La evaluación funcional de pacientes psiquiátricos tras 12 meses de incapacidad temporal detecta ausencia de incapacidad en el 46,39\%, generalmente ligado al trastorno adaptativo. Las escalas psicométricas son capaces de señalar discapacidad. Se han detectado marcadores de gravedad y disfunción como son la vivencia de apoyo social, la ausencia de pareja, el tiempo en seguimiento psiquiátrico, los antecedentes familiares psiquiátricos y las escalas psicométricas. Existe una alta comorbilidad física y un tabaquismo preocupante en los pacientes psiquiátricos. Son necesarios estudios similares que puedan confirmar o perfilar los datos aquí presentados.

Med Segur Trab (Internet) 2013; 59 (231) 205-226

Palabras clave: Incapacidad temporal de causa psiquiátrica, evaluación funcional psiquiátrica, incapacidad temporal de larga duración, apoyo social.

Abstract

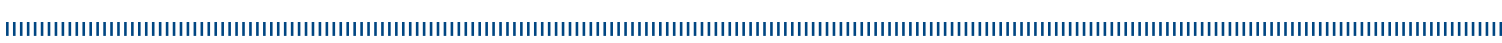

Objective: Psychiatric Pathology is a major problem because it is the main cause of loss of quality of life while it generates large individual, social and economic costs. Despite the mentioned above, few data are available on the clinical features of psychiatric work disabled patients. We aim to describe the mentioned features in patients with temporary disability for more than 12 months and to investigate the factors associated with chronicity or disability.

Metbods: A total of 97 patients with temporary disability for more than 12 months were selected from all of the patients referred to our office by the INSS for psychiatric evaluation. Main clinical, social and labour features were recorded in addition to the diagnosis, according to DSM-IV-TR criteria. Global Assessment of Functioning (GAF) and Symptom Intensity scales (MADRS and WHODAS O) were used.

Results: Our sample had a balanced sex ratio and an average age of 47 years. Patients usually described an experience of moderate social support and 51.5\% of them were living as a couple. A third of the patients had primary education at most. A noteworthy physical illness background was found in $78.4 \%$ of them, 50.5\% had smoking habits and 59\% had family history of psychiatric disorders. All of these conditions tend to be associated with low functionality and longer treatments. We have found a $71.1 \%$ diagnostic agreement and the most frequent diagnosis-related group were affective disorders. In the group of patients without functional limitation, adjustment disorder was the most frequent diagnosis. It was associated to shorter treatments and lower levels of Symptom Intensity. The scales that we used correlated with each other. GAF and WHODAS $\mathrm{O}$ distinguish adjustment disorders in non-disabled patients while MADRS points out affective disorders. Decisions made after assessment were: no disability in $46.39 \%$ of the patients, grant an extension of paid sick leave days in $11.34 \%$, permanent occupational disability to work in their previous job in $29.90 \%$ and permanent occupational disability to work in any job in $12.37 \%$. Patients with permanent occupational disability were associated with low social support, prolonged psychiatric follow-up, family history of psychiatric disorders and diagnosis other than adjustment disorder.

Conclusions: The functional assessment of psychiatric patients after a 12-month period of temporary disability showed no disability in $46.39 \%$ of the patients and it is usually bound to adjustment disorder. Psychometric scales may mark occupational disability.

Markers of severity and dysfunction have been identified like social support experience, absence of a partner, psychiatric follow-up span, family history of psychiatric disorders and psychometric scales. Physical comorbidity is highly frequent and smoking habits are disturbing among psychiatric patients. Similar studies are needed to confirm our results and increase knowledge on this subject.

Med Segur Trab (Internet) 2013; 59 (231) 205-226

Key words: Temporary disability by psychiatric illness, psychiatric functional evaluation, long-term work disability, social support. 


\section{INTRODUCCIÓN}

Estudios recientes realizados en Europa y EEUU sitúan la patología psiquiátrica como la primera causa de pérdida de la calidad de vida expresada como DALY (Años de Vida Ajustados a Discapacidad) ${ }^{1,2}$ y como el principal motivo de solicitud de pensión por incapacidad. ${ }^{3}$ En sólo 1 año, una de cada tres personas presentará síntomas suficientes para cumplimentar los criterios diagnósticos de alguna patología psiquiátrica. ${ }^{1}$

La incapacidad laboral sobrevenida genera grandes costes personales y económicos. Estos últimos se estimaron para 2010 en la UE (Unión Europea) en 798.000 millones de $€$, de los cuales el $40 \%$ corresponde a costes indirectos ligados a la pérdida de productividad. ${ }^{4}$ El coste económico de los principales grupos de patología psiquiátrica se muestra en la tabla 1. Algunos estudios señalan que si se aumentara el gasto en las partidas de tratamiento, se podría disminuir las partidas indirectas, especialmente las ligadas a la pérdida de la capacidad, especialmente en el caso de la ansiedad y de la depresión. ${ }^{5}$

Tabla 1. Costes económicos de los principales grupos de patología psiquiátrica en la Unión Europea en 2010 (expresado en Miles de Millones de $€)^{4}$

\begin{tabular}{lc}
\hline \multicolumn{1}{c}{ Grupos Psiquiátricos } & Costes económicos \\
\hline Trastornos Afectivos & $113,4 \mathrm{MM}$ \\
Trastornos Psicóticos & $93,9 \mathrm{MM}$ \\
Trastornos de Ansiedad & $74,4 \mathrm{MM} €$ \\
Trastornos por Adicción & $65,7 \mathrm{MM} €$ \\
Retraso Mental & $43,3 \mathrm{MM}$ \\
Trastornos de la Personalidad & $27,2 \mathrm{MM} €$ \\
Trastornos Somatomorfos & $21,2 \mathrm{MM}$ \\
\hline
\end{tabular}

El cálculo de los costes económicos de cualquier patología conlleva sus peculiaridades y dificultades, con el riesgo de la infra o supravaloración. Se puede caer en la infravaloración por la imposibilidad de acceso a los costes indirectos, ${ }^{6-8}$ como los asociados a IP (Incapacidad Permanente), ${ }^{9}$ o por la dificultad diagnóstica en pacientes que se pudieran presentar bajo diagnósticos físicos. ${ }^{10}$

Pese al comentario popular de que se está realizando un sobrediagnóstico psiquiátrico, una psiquiatrización de la vida cotidiana, estudios consistentes realizados en el ámbito europeo, denotan lo contrario. En poblaciones que han experimentado la guerra, como en Bosnia, ${ }^{11}$ sí que se ha producido un aumento del número de casos, pero también mayor gravedad en las patologías afectivas expresada en el porcentaje de pensiones concedidas, mientras que las patologías psicóticas se mantienen en los números previos. En el resto de Europa,${ }^{1}$ sin guerras recientes, se presenta una estabilidad en los datos, de forma que desde 2005 no hay incremento porcentual de casos, considerándose previo a ello una situación de infradiagnóstico.

Esta dificultad diagnóstica queda señalada en el estudio de Araña ${ }^{12}$, tanto a nivel internacional como nacional. Al médico de atención primaria le cuesta realizar un diagnóstico psiquiátrico con la deseable especifidad y fijar unos criterios óptimos para el manejo de la IT (Incapacidad Temporal) por causa psiquiátrica.

En España, la patología psiquiátrica constituye el segundo grupo en frecuencia, tras la patología osteomuscular, y también el segundo grupo en duración, tras la oncológica. ${ }^{13}$ Para disminuir la ITCC (Incapacidad Temporal por Contingencia Común) de causa psiquiátrica se realiza la derivación al psiquiatra. Cuando no es posible en plazos razonables en el SPS (Sistema Público de Salud), es frecuente que las mutuas recurran a consultores externos. De esta forma se consigue disminuir el tiempo en IT cuando la derivación se realiza precozmente, pero si el fin de esta derivación es generar informes para la petición del alta no se consigue disminuir la IT. ${ }^{14}$ 
Los datos del INSS (Instituto Nacional de la Seguridad Social) respecto a la IT mayor de 12 meses en 2009 en España, ${ }^{15}$ indican que casi la mitad de los casos psiquiátricos pasan a prórroga ( 1 de cada 7 prórrogas, 13,63\%, son de causa psiquiátrica) y la mitad de los casos psiquiátricos son dados de alta (1 de cada 5 altas, $18.99 \%$ son de causa psiquiátrica), con la distribución por patologías principales que se observa en la tabla 2.

Tabla 2. Porcentajes de patologías psiquiátricas tras ITCC $>12$ meses ${ }^{15}$

\begin{tabular}{lcc}
\hline & Prórroga tras ITCC $>\mathbf{1 2 m}$ & Alta tras ITCC $>\mathbf{1 2 m}$ \\
\hline Trastorno Depresivo & $5,09 \%$ & $5,29 \%$ \\
Trastorno Adaptativo & $1,99 \%$ & $3,10 \%$ \\
Trastornos de Ansiedad & $6,55 \%$ & $10,60 \%$ \\
\hline
\end{tabular}

Datos similares se obtienen en las evaluaciones realizadas por el INSS en Valencia, ${ }^{16}$ donde la patología psiquiátrica representa el segundo grupo en frecuencia en las evaluaciones a 12 meses, alcanzando la IP el 48,03\% de los pacientes valorados. En dicha muestra, la edad media se situaba en los 50,96 años, los varones suponían un 59,36\% frente al 40,64\% de las mujeres, destaca en el grupo a valorar para incapacidad permanente que el $61,14 \%$ de los pacientes tenían estudios que no superaban la primaria.

La IP ha supuesto para el INSS ${ }^{17}$ en 2011 un desembolso de 823 millones de $€$, de la cual se estima que cerca del $30 \%$ son de causa psiquiátrica. Las características demográficas principales de esta población son una moda de edad entre 55 y 60 años con una relación de 2 varones por cada mujer. Probablemente este peso creciente en el coste económico de la incapacidad laboral ha llevado al INSS a casi duplicar su plantilla facultativa experta en evaluación desde 2005 (371 médicos) a 2010 (633 médicos), ${ }^{15}$ pese a lo cual siguen sin contar con especialistas en psiquiatría, por lo que para dichas valoraciones, cuando son complejas, se realizan por especialistas de otras entidades.

Uno de los problemas clásicos con la IT psiquiátrica es que conforme más avanza el tiempo en IT, mayor es la probabilidad de no volver al trabajo, a partir de los 6 meses sólo lo hace el $50 \%$, proporción que empeora con la edad o en el género masculino. ${ }^{18}$ Por ello se han realizado múltiples estudios tratando de señalar factores predictores sobre la ITCC de larga duración en general ${ }^{19}$ y en la patología psiquiátrica. ${ }^{20-22}$ En general no son completamente coincidentes y suelen encontrar hallazgos que parecen estar ligados a la psicobiografía socialmente discapacitante de estas enfermedades, por lo que los iremos analizando durante la discusión de los resultados.

Por todo ello nos proponemos en el siguiente estudio analizar las características clínicas y sociolaborales de las personas con patología psiquiátrica en situación de IT prolongada, realizar su descripción e investigar factores pronósticos que pudieran servir de marcadores de cronicidad y de capacidad funcional para facilitar el trabajo evaluador y abrir nuevos campos de investigación.

\section{MATERIAL Y MÉTODOS}

Para la realización del estudió se decidió incluir en él a todos los pacientes derivados desde el INSS de Madrid para evaluación psiquiátrica especializada, orientada fundamentalmente a los aspectos diagnósticos y funcionales. Este grupo estaba compuesto por 109 individuos, pero se componía de 2 subgrupos. El primero estaba formado por 97 individuos que habían superado los 12 meses de ITCC y se encontraban pendientes de resolución hacia alta laboral, prórroga o demora de calificación, e inicio de expediente de IP. El segundo subgrupo, de 12 individuos, lo constituían los pacientes que ya disponían de la IP y eran derivados dentro de su proceso de revisión de grado. Éstos presentan unas características clínicas y funcionales distintas de los primeros, por lo que se consideró criterio de exclusión la concesión previa de IP y el grupo definitivo de estudio quedó 
constituido por los 97 pacientes del grupo primero. De esta forma se estructuró este estudio descriptivo transversal tras evaluación única.

La evaluación de todos los pacientes se realizó mediante la aplicación de un protocolo de exploración psicopatológica semiestructurada, con recogida de las principales variables sociodemográficas (edad, sexo, convivencia, número de hijos nacidos y a su cargo, mayores dependientes a su cargo, vivencia de apoyo social, conflictos familiares o laborales, nivel de estudios y profesión última) y variables clínicas (antecedentes personales somáticos, tabaquismo, alcoholismo, otras toxicomanías, antecedentes familiares psiquiátricos, seguimiento en el SPS, años en seguimiento psiquiátrico, diagnóstico referido del INSS, diagnósticos principales y secundarios realizados en la evaluación, y concordancia diagnóstica entre el diagnóstico referido desde el INSS y el hallado en consulta.

La evaluación del nivel de estudios se realizó considerando el ciclo formativo completado, con los siguientes grupos: los que no terminaron estudios primarios, los que culminaron los estudios primarios, los que completaron estudios secundarios o equivalentes, y los que terminaron algún estudio universitario. Este tipo de clasificación ha mostrado asociación con la capacidad de las personas para resolver conflictos y con los sueldos recibidos. ${ }^{23}$

La profesión, aunque no se dispuso del código exacto durante la entrevista, fue clasificada posteriormente según la descripción del paciente de su puesto específico y sus requerimientos, en función de las fichas ocupacionales de Ibermutuamur y la Clasificación Nacional de Ocupaciones (CNO-2011). ${ }^{24} \mathrm{El}$ análisis individualizado por profesiones requiere unas muestras de gran tamaño para obtener representación estadísticamente suficiente en cada subgrupo, por lo que se decidió agrupar dichas profesiones en sus grupos principales, como suele acabar siendo habitual en los trabajos de la medicina ocupacional. ${ }^{18,25-28}$

Los diagnósticos fueron realizados de acuerdo con los criterios diagnósticos de la clasificación DSM-IV-TR ${ }^{29}$ con la codificación asignada a la clasificación en uso por la administración pública española, que es la CIE-9-MC. ${ }^{30}$ De la misma forma que con las profesiones, los diagnósticos fueron agrupados para su estudio en grupos similares de comportamiento clínico, funcional y etiopatogénico de la siguiente forma: los que no se detectaba patología psiquiátrica, trastornos adaptativos, trastornos del eje II de la DSM-IV-TR (trastornos de personalidad y retraso mental), trastornos afectivos, trastornos psicóticos, trastornos de ansiedad, trastornos por adicción a sustancias, y un grupo final con otras patologías psiquiátricas.

Asimismo se aplicaron la Escala de Evaluación de la Actividad Global (EEAG), ${ }^{29}$ de intensidad sintomatológica como la escala de depresión de Montgomery Asberg (MADRS) ${ }^{31}$ o la escala de sintomatología positiva y negativa en la esquizofrenia (PANSS), ${ }^{31}$ y de discapacidad como la escala de discapacidad de la OMS (WHODAS) ${ }^{31}$ completa, si bien seleccionamos la subescala ocupacional (WHODAS-O) por el medio laboral en el que nos encontramos. Finalmente se registró la propuesta orientadora para el INSS, entendida como enfermedad psiquiátrica no incapacitante, enfermedad psiquiátrica incapacitante en el momento actual susceptible de mejora suficiente en 2-6 meses, enfermedad psiquiátrica incapacitante para su profesión o enfermedad psiquiátrica incapacitante para cualquier profesión.

Se constituyó una base de datos para el programa estadístico SPSS en su versión 2.0. Para las descripción de las medidas cuantitativas se utilizaron medias, mediana y moda en distribuciones asimétricas, así como la desviación estándar, su representación se ha realizado en histogramas. Las variables cualitativas, nominales u ordinales, se han descrito a través de su distribución porcentual, con representación en gráficos de columnas o de sectores. Para la estadística inferencial se ha utilizado la prueba T para comparación de medias entre variables cuantitativas y cualitativas, U de Mann-Whitney para variables ordinales y cualitativas independientes de 2 categorías, la prueba de Kruskal-Wallis para variables ordinales y cualitativas independientes de más de 2 categorías, la $\mathrm{X}^{2}$ para las 
variables cualitativas independientes entre sí, la prueba de Pearson para la correlación entre variables cuantitativas y la rho de Spearman para variables cuantitativas y ordinales. El conjunto de datos se trató conforme a la declaración de Helsinki, ${ }^{32}$ asegurando la privacidad de los pacientes en el tratamiento de sus datos.

Entre las limitaciones del presente estudio está la imposibilidad estadística de profundizar individualizadamente en algunas patologías concretas, e incluso en los grupos patológicos menos frecuentes, al igual que sucede con las profesiones, limitándonos a su análisis por grandes grupos.

Otra posible limitación es la interpretación de la derivación a evaluación especializada como sesgo de selección. Los pacientes evaluados no son el total de pacientes psiquiátricos del INSS de Madrid, ni siquiera una proporción aleatoria y fidedigna. Se trata probablemente de casos complejos que generan más dudas y son de especial dificultad para la evaluación.

Asimismo, los datos presentados no se han podido relacionar con las resoluciones del INSS o con datos económicos de los trabajadores, como las bases reguladoras, de gran interés para este tipo de estudios, quedando pendientes de incorporarse en futuros estudios.

\section{RESULTADOS}

Las características sociodemográficas de la muestra aportan una distribución por géneros homogénea (gráfico 1), con una edad media de 47,02 y DE=10,33, un rango entre 21 y 63 y una moda de 50 (gráfico 2). No se aprecian diferencias entre los distintos grupos clínicos. En el gráfico 3 se puede observar la pirámide poblacional por sexos, sin diferencias significativas en su distribución por edades en el grupo general.

Gráfico 1. Distribución por sexos



En cuanto al tipo de convivencia, el 51,5\% vive con pareja, frente al resto, que lo hace solo, en viviendas compartidas o con la familia de origen. Cuando se analiza por patologías (gráfico 4), resulta que sólo el 16,7\% del grupo psicótico vive en pareja $\left(\mathrm{X}^{2}=84,67 ; \mathrm{P}<0,001\right)$, extremo contrario al grupo depresivo, que vive en pareja el $61 \%$, $\left(X^{2}=13,81 ; P<0,001\right)$.

El 58,8\% de los pacientes tiene hijos, con una media de 1,98, DE=0,876, moda en 2 y rango entre 1 y 4 . El 41,2\% de la muestra tiene hijos a su cargo, con una media de 1,75, $\mathrm{DE}=0,707$, moda de 2 y rango entre 1 y 4 . Al analizar otras cargas familiares, como el cuidado de personas mayores dependientes, sólo el 6,2\% de los pacientes son responsables de un ascendiente dificultado, sin asociarse a ningún grupo diagnóstico.

Menos de un tercio de los pacientes sienten un apoyo social positivo, y analizado por grupos diagnósticos se aprecian diferencias entre el apoyo social nulo o limitado a la familia y el positivo (gráfico 5). Considerado el apoyo social como una variable ordinal, se aprecia diferencia significativa entre hombres y mujeres $(U=847,0 ; p=0,012)$. Otras significaciones se señalan más adelante. 
Gráfico 2. Distribución por edad

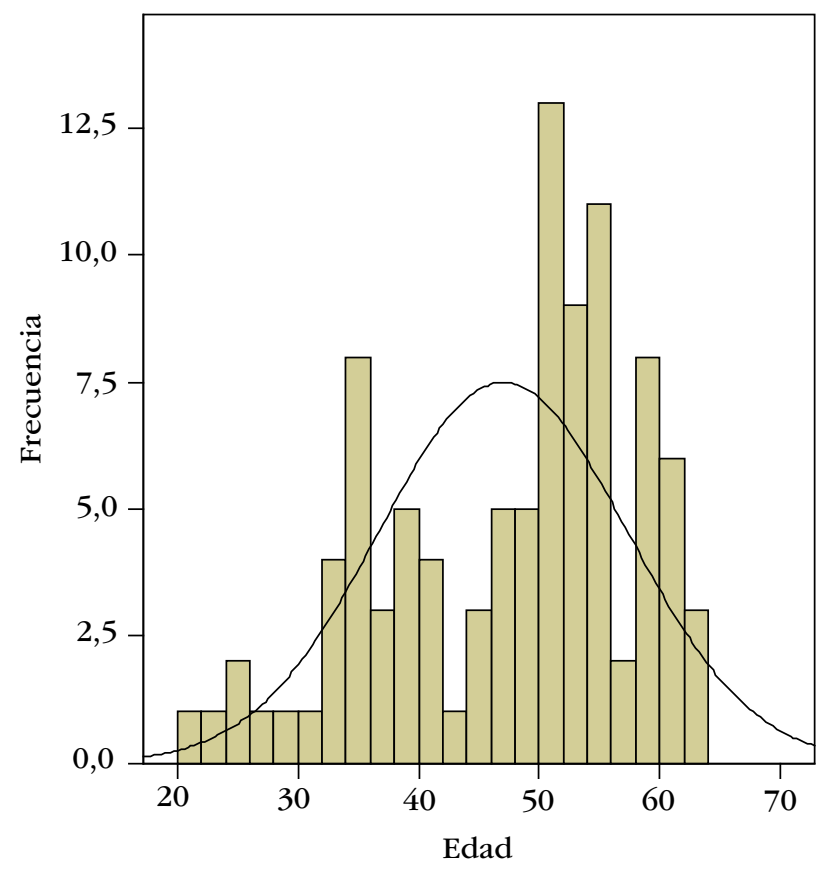

Gráfico 3. Pirámide poblacional de ITCC $>12$ meses

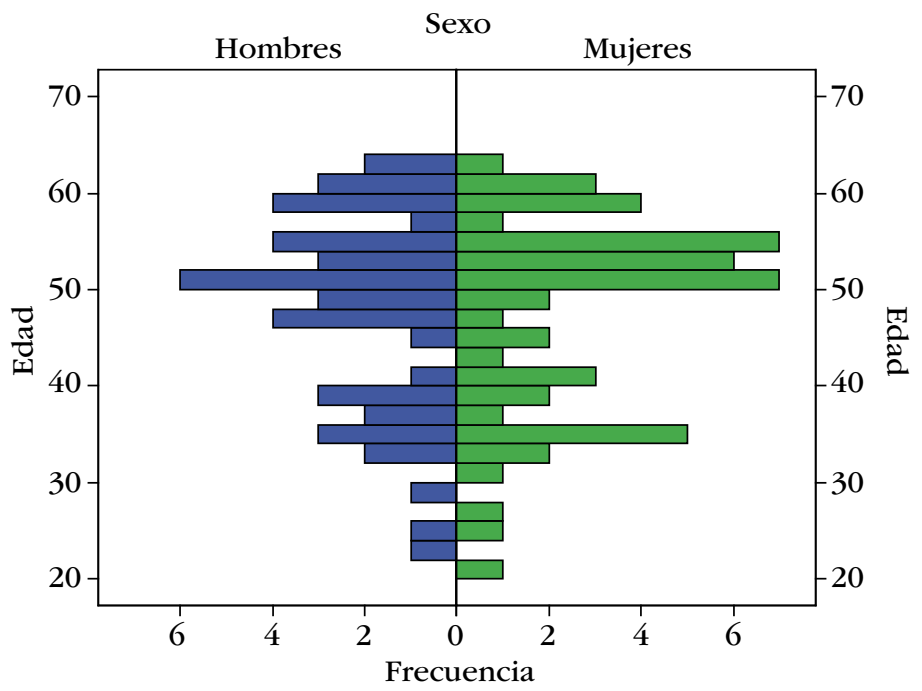

Gráfico 4. Tipo de convivencia por grupos patológicos

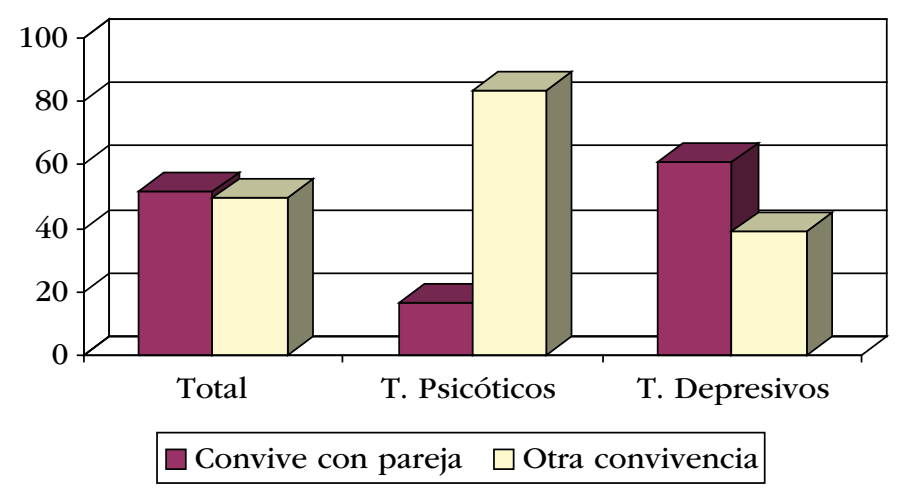


Gráfico 5. Apoyo social total, por grupos diagnósticos y por sexos

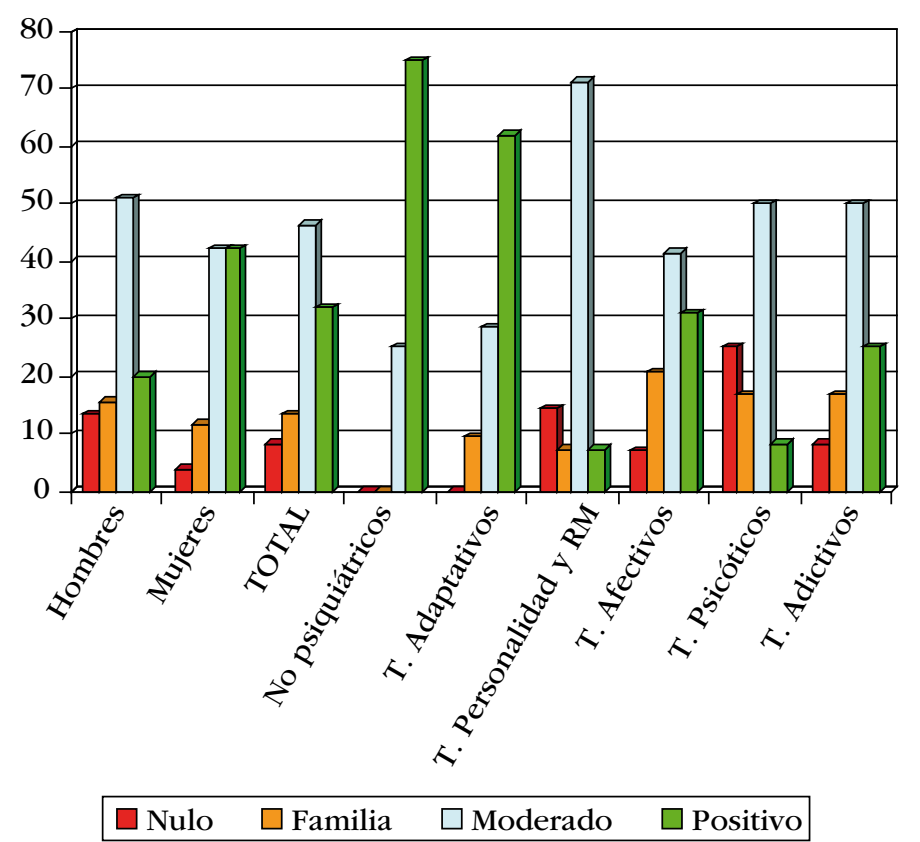

La presencia de conflicto familiar en el $13,4 \%$ de la muestra no se asoció a ninguna otra variable. Sin embargo, la vivencia de conflicto laboral, presente en el 19,6\%, se sobrerrepresenta de forma significativa en mujeres en el 78,9\% ( $\left.X^{2}=4,908 ; p=0,027\right)$, es más frecuente en personas con menos hijos $(1,53 ; \mathrm{DE}=0,743)$, con mayor formación superada ( $U=523,50 ; p=0,042)$, y menor puntuación en la escala depresiva (18,06; $\mathrm{DE}=10,152)$. El conflicto laboral está con más frecuencia, el 38,1\%, en los cuadros adaptativos de forma estadísticamente significativa $\left(X^{2}=4,428 ; p=0,035\right)$.

La distribución del grado de formación superado se muestra en el gráfico 6 y su relación con otras variables clínicas se comentará con ellas. La CNO-2011 presenta una distribución que mostramos en el gráfico 7 y que no presenta diferencias por sexos ni asociación o tendencia con ninguno de los valores estudiados.

Gráfico 6. Formación superada

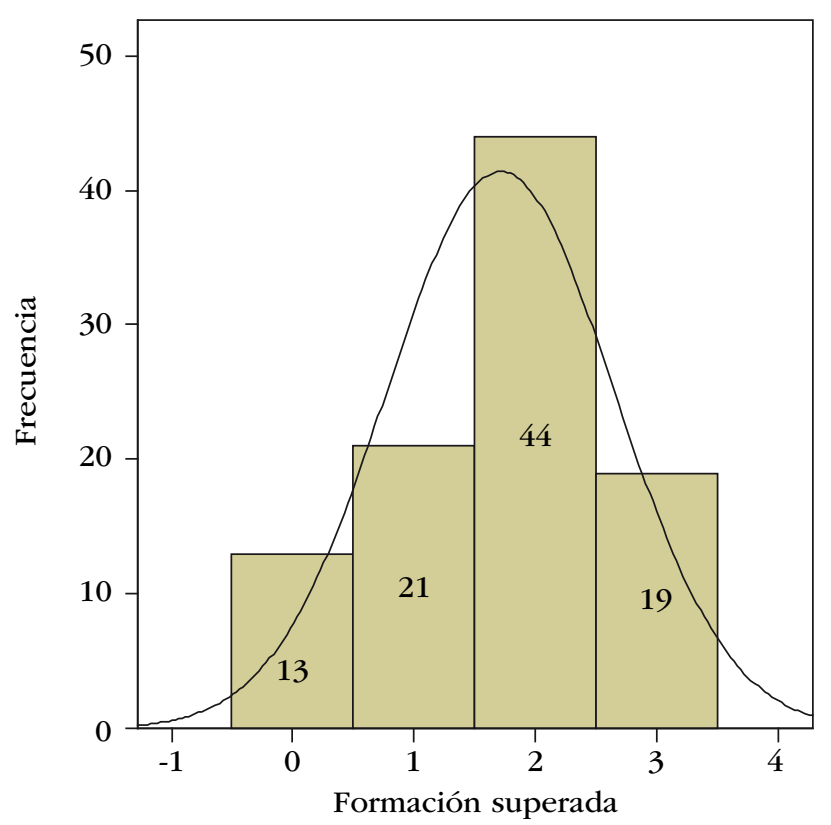


Gráfico 7. Grupos de la CNO-11

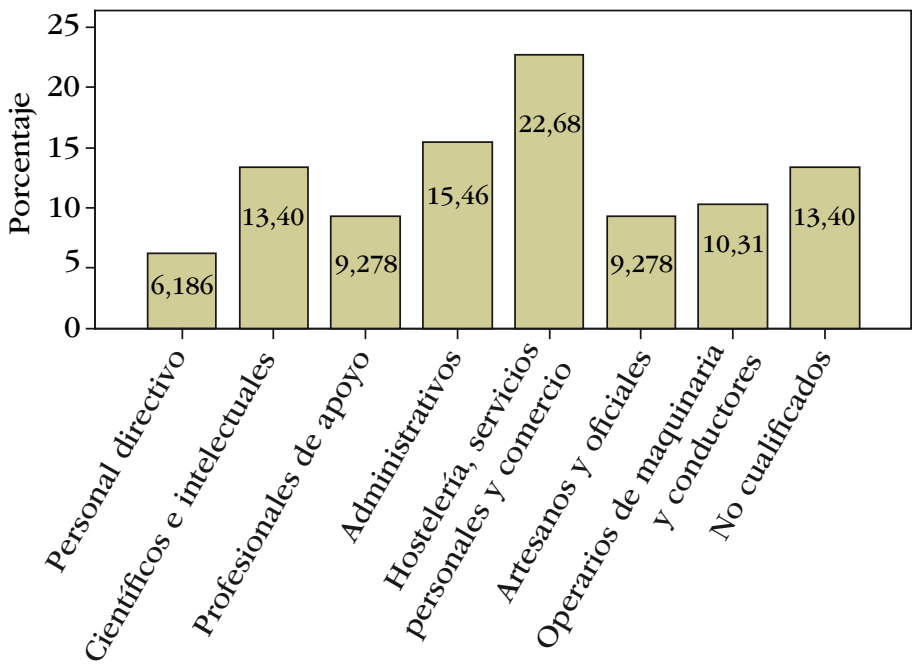

En los aspectos clínicos de la muestra el $78,4 \%$ de la población remitida a estudio cuenta con antecedentes físicos relevantes y presentan asociación con mayor edad (49,32 años, DE=9,078; frente a 38,71 años, $\mathrm{DE}=10,536 ; \mathrm{t}=4,200 ; \mathrm{p}<0,001)$, con mayor número de hijos tenidos $(2,13$ frente a 1,$30 ; \mathrm{t}=4,158 ; \mathrm{p}<0,001)$ e hijos a cargo $(1,88$ frente a 1,$25 ; \mathrm{t}=3,035 ; \mathrm{p}=0,008)$, así como de forma inversa con el grado de formación superada $(\mathrm{U}=565,0 ; \mathrm{p}=0,030)$.

El consumo de tabaco presenta así mismo un grave porcentaje, el 50,5\%, con una media de 18,84, mediana y moda en 20 y $\mathrm{DE}=12,85$. De forma curiosa, aparece una correlación positiva entre el número de hijos tenidos $(r=0,409 ; \mathrm{p}=0,028)$ y con el número de hijos a cargo $(\mathrm{r}=0,473 ; \mathrm{p}=0,030)$. Aunque en el grupo de los trastorno ansiosos hay un mayor número de hijos como media $(2,25 ; \mathrm{DE}=0,707)$, no presenta diferencia significativa, como tampoco sucede entre los distintos grupos de diagnóstico y el consumo de tabaco.

El alcoholismo se encuentra presente en un 18,6\% de la muestra y se asocia con los años en seguimiento psiquiátrico, 12,33 años y $\mathrm{DE}=11,956$ frente a 6,52 años y $\mathrm{DE}=7,060$, $(\mathrm{t}=2,730 ; \mathrm{p}=0,008)$. Otras toxicomanías se encuentran presentes en un $7,2 \%$.

Los antecedentes familiares psiquiátricos, presentes en el 59,8\%, se asocian con otras variables como los años en tratamiento psiquiátrico $(t=2,158 ; \mathrm{p}=0,033)$ y el número de diagnósticos psiquiátricos $(\mathrm{t}=2,124 ; \mathrm{p}=0,036)$, además de las escalas psicométricas empleadas (EEAG, MADRS, WHODAS O) que se analizan más adelante.

La media de tiempo en tratamiento psiquiátrico se sitúa en 7,60 años, $\mathrm{DE}=8,424$ en un rango entre 0 y 45, con una mediana de 5 y una moda de 1 (gráfico 8). Presentan correlación negativa de forma significativa $(r=-0,252 ; p=0,013)$ con la EEAG, cuantos más años en tratamiento psiquiátrico, más se reduce la actividad global de los pacientes. Otras asociaciones se comentarán más adelante.

La agrupación de los diagnósticos referidos en la derivación del INSS en categorías principales se muestran con sus valores en el gráfico 9 y los realizados con criterios DSM-IV-TR tras la evaluación psiquiátrica en el gráfico 10. Sólo hay discrepancia diagnóstica en el $28,9 \%$ de los casos, mientras que existe coincidencia diagnóstica en el $71,1 \%$ en el grupo diagnóstico, y de forma plena en el código exacto, no solo en el grupo diagnóstico, en el 33,0\%.

Analizadas las características del trastorno adaptativo, se observa junto a los que no presentan patología, una gran diferencia significativa $\left(X^{2}=8,956 ; p=0,030\right)$ en el sentimiento de apoyo social frente al resto de diagnósticos psiquiátricos. Estos grupos (adaptativos y sin diagnóstico frente a los demás) también se diferencian en tener menor número de diagnósticos psiquiátricos, 0,96 frente a 1,49 ( $\mathrm{t}=3,634 ; \mathrm{p}<0,001)$, y menor 
número de años en seguimiento psiquiátrico 3,24 frente a 9,11 ( $\mathrm{t}=3,137, \mathrm{p}=0,002)$ como colectivo y frente a los trastornos afectivos, los trastornos psicóticos y los adictivos. En cuanto a las escalas de intensidad sintomatológica y situación funcional, también presentan diferencias significativas que comentaremos más adelante.

Gráfico 8. Años en tratamiento psiquiátrico

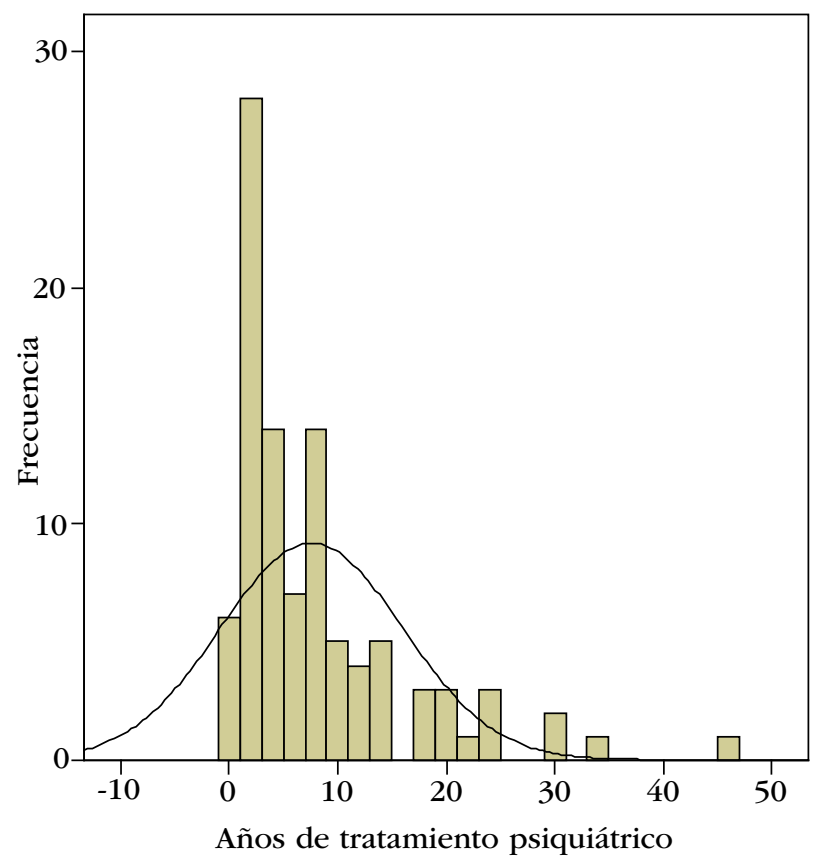

Gráfico 9. Diagnósticos Grupales según INSS

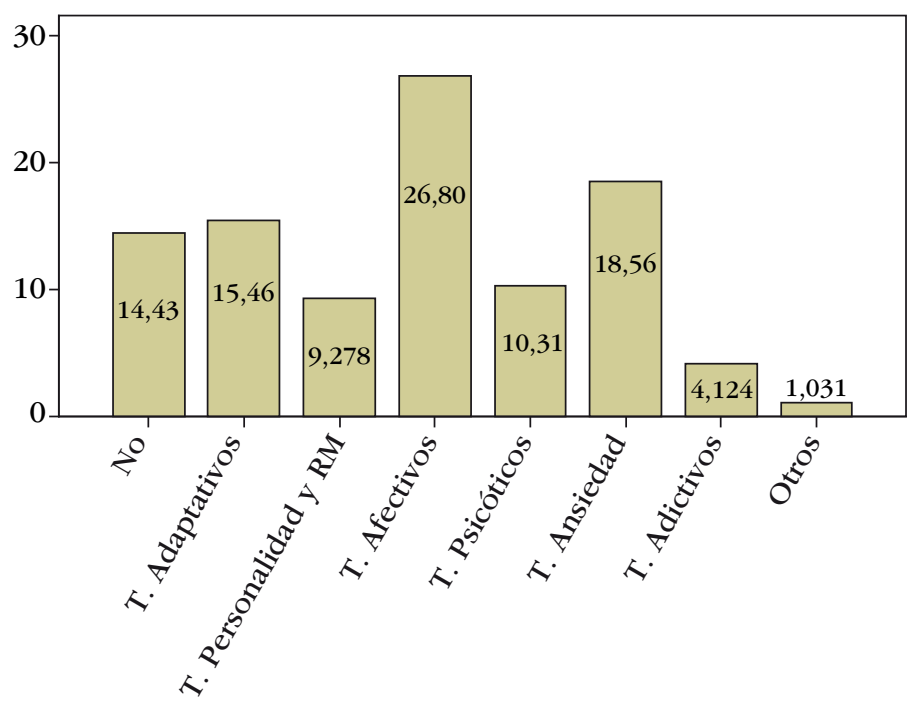

La comorbilidad psiquiátrica en nuestra muestra presenta de forma significativa únicamente la diferencia ya comentada entre el grupo de los trastornos adaptativos y los demás diagnósticos. Un segundo diagnóstico sucede en el $26,8 \%$, con la distribución presentada en la tabla 3. El tercer diagnóstico sucede sólo en el 6,2\% y se presenta en la tabla 4 .

Las escalas psicométricas utilizadas aportan resultados muy interesantes e intensas correlaciones entre sí (tabla 5). La EEAG presenta una puntuación media de 55,47, $\mathrm{DE}=11,9632$ y un rango entre 25 y 90 , con una distribución totalmente normalizada que observamos en el gráfico 11. Analizada esta escala por diagnósticos y por propuestas de 
capacidad funcional realizada, se obtienen diferencias, significativas $(t=-5,984 ; \mathrm{p}<0,001)$ como bloque o individualmente entre el grupo de los trastornos adaptativos y los demás trastornos psiquiátricos, excepto las adicciones. Asimismo se obtiene diferencia significativa entre los que se les propone para algún grado de incapacidad y los que no se les considera incapacitados o son recuperables funcionalmente en el tiempo de prórroga $(\mathrm{t}=-8,692, \mathrm{p}<0,001)$. Se presentan las medias en el gráfico 12.

\section{Gráfico 10. Diagnósticos Grupales Principales}

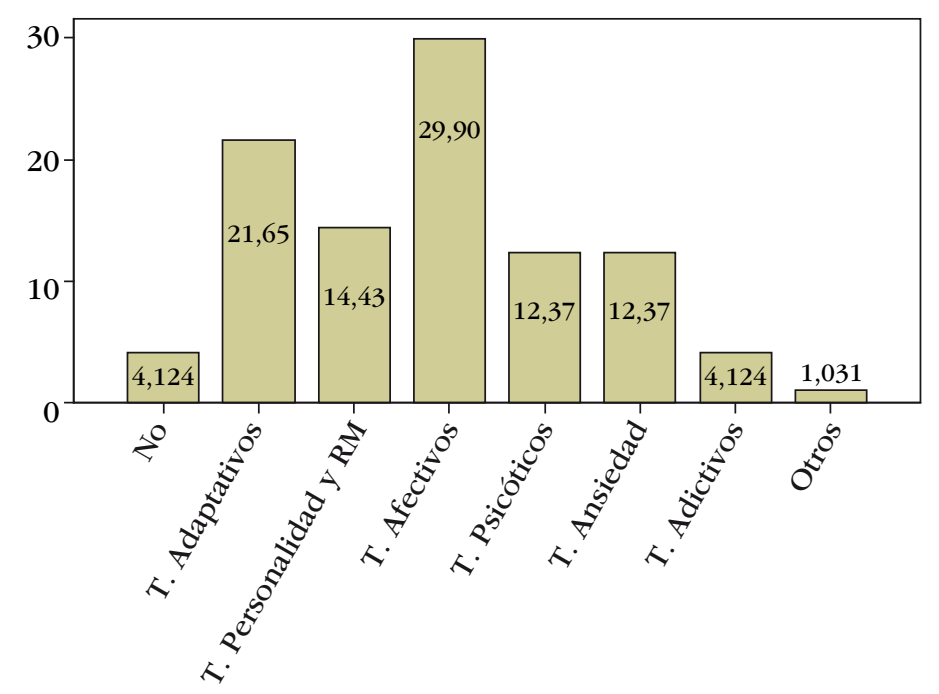

Tabla 3. Diagnóstico Grupal 2.

\begin{tabular}{llccc}
\hline & & Frecuencia & Porcentaje total & Porcentaje grupal \\
\hline \multirow{2}{*}{ Presentes } & T. Personalidad y RM & 13 & 13,4 & 40,6 \\
& T. Afectivos & 7 & 7,2 & 21,9 \\
& T. Psicóticos & 1 & 1,0 & 3,1 \\
& T. Ansiedad & 4 & 4,1 & 12,5 \\
& T. Adictivos & 5 & 5,2 & 15,6 \\
& Otros & 2 & 2,1 & 6,3 \\
Ausentes & Total & 32 & 33,0 & 100,0 \\
Total & & 65 & 67,0 & \\
\hline
\end{tabular}

Tabla 4. Diagnóstico Grupal 3.

\begin{tabular}{llccc}
\hline & & Frecuencia & Porcentaje total & Porcentaje grupal \\
\hline Presentes & T. Personalidad y RM & 2 & 2,1 & 33,3 \\
& T. Afectivos & 2 & 2,1 & 33,3 \\
& T. Adictivos & 1 & 1,0 & 16,7 \\
& Otros & 1 & 1,0 & 16,7 \\
& Total & 6 & 6,2 & 100,0 \\
Ausentes & & 91 & 93,8 & \\
Total & & 97 & 100,0 & \\
\hline
\end{tabular}

La escala de depresión de Montgomery Asberg (MADRS), presenta una media de 23,57, lo que corresponde a sintomatología moderada, con $\mathrm{DE}=9,672$ y un rango entre 3 y 41 . También muestra diferencias entre el grupo afectivo y los demás $(\mathrm{t}=2,331 ; \mathrm{p}=0,033)$ y entre los que se les propone IP y los que no $(\mathrm{t}=2,886 ; \mathrm{p}=0,005)$, como observamos en el gráfico 13 . 
Tabla 5. Correlaciones significativas entre las escalas empleadas

\begin{tabular}{llrrr}
\hline & & EEAG & MADRS & WHODAS O \\
\hline \multirow{2}{*}{ EEAG } & Correlación de Pearson & 1 & $-0,525^{*}$ & $-0,751^{*}$ \\
& Sig. (bilateral) & & 0,000 & 0,000 \\
& $\mathrm{~N}$ & 96 & 84 & 95 \\
\multirow{3}{*}{ MADRS } & Correlación de Pearson & $-0,525^{*}$ & 1 & $0,496^{*}$ \\
& Sig. (bilateral) & 0,000 & & 0,000 \\
& $\mathrm{~N}$ & 84 & 84 & 83 \\
\multirow{3}{*}{ WHODAS O } & Correlación de Pearson & $-0,751^{*}$ & $0,496^{*}$ & 1 \\
& Sig. (bilateral) & 0,000 & 0,000 & \\
& $\mathrm{~N}$ & 95 & 83 & 95 \\
\hline
\end{tabular}

* La correlación es significativa al nivel 0,01 (bilateral).

Gráfico 11. EEAG

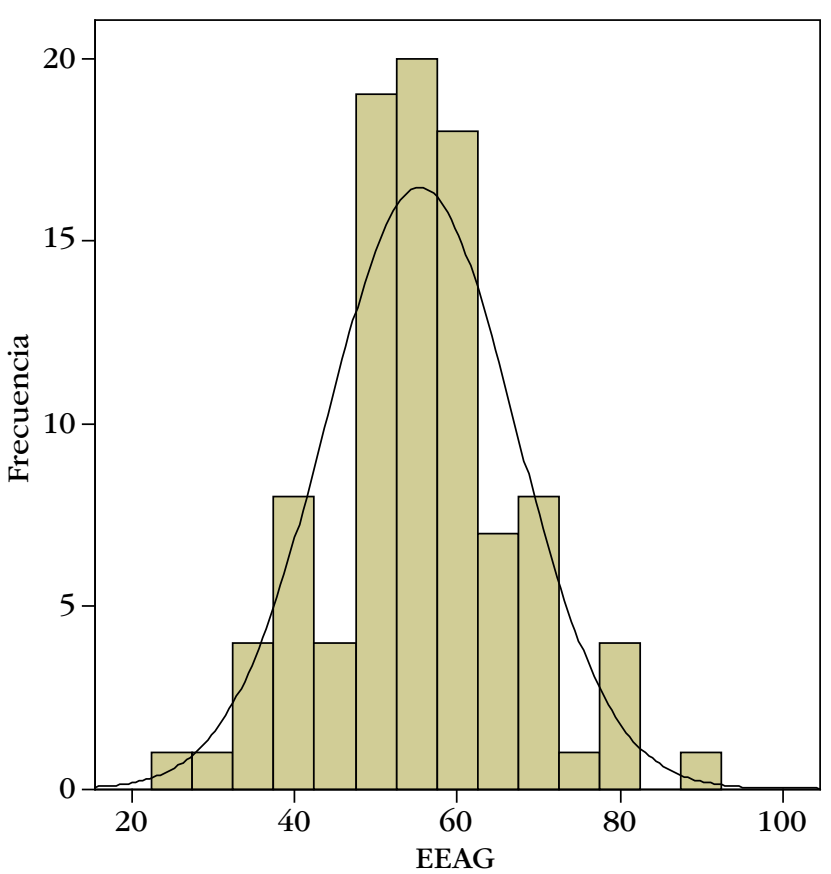

Gráfico 12. EEAG por patologías y propuestas

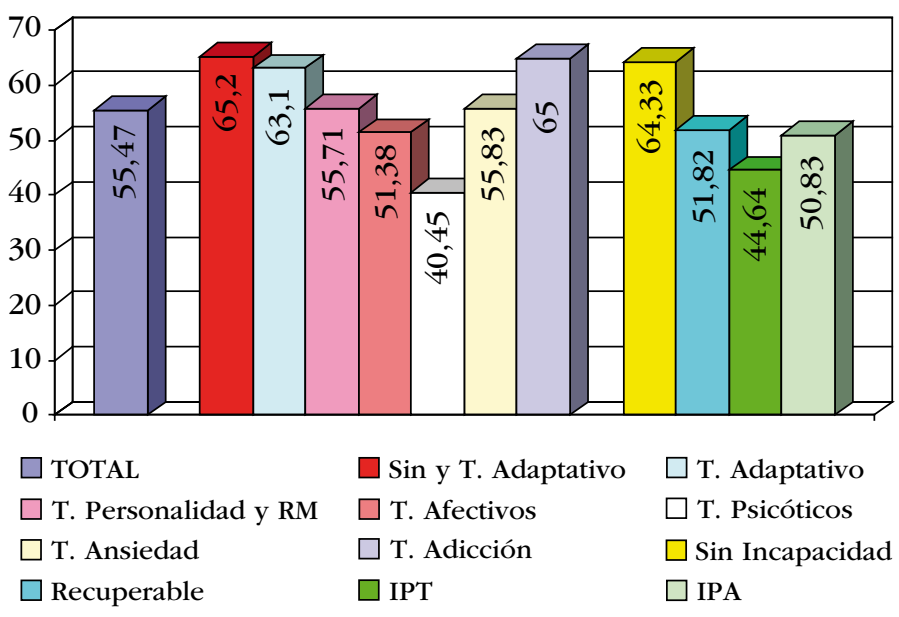


Gráfico 13. MADRS por patologías y propuestas

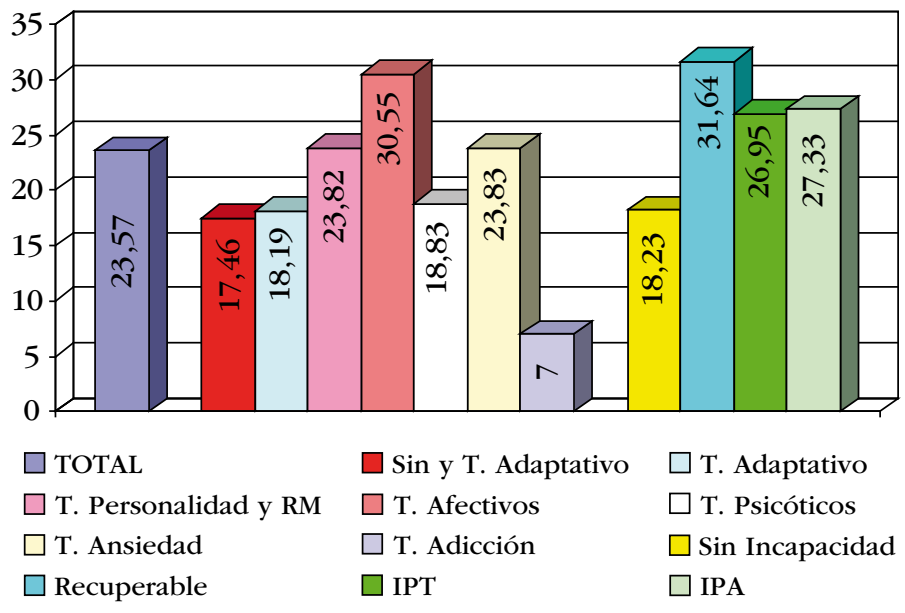

La escala de discapacidad de la OMS en la subescala ocupacional, WHODAS O (gráfico 14) presenta una media de $2,57, \mathrm{DE}=1,108$ con una distribución variable por patologías, en especial entre adaptativos y los demás $(\mathrm{t}=6,637 ; \mathrm{p}<0,001)$, excepto adicciones, y entre los que no se les encuentra incapacitación en la evaluación y los demás ( $\mathrm{t}=9,694 ; \mathrm{p}<0,001)$.

Gráfico 14. WHODAS O por patologías y propuestas

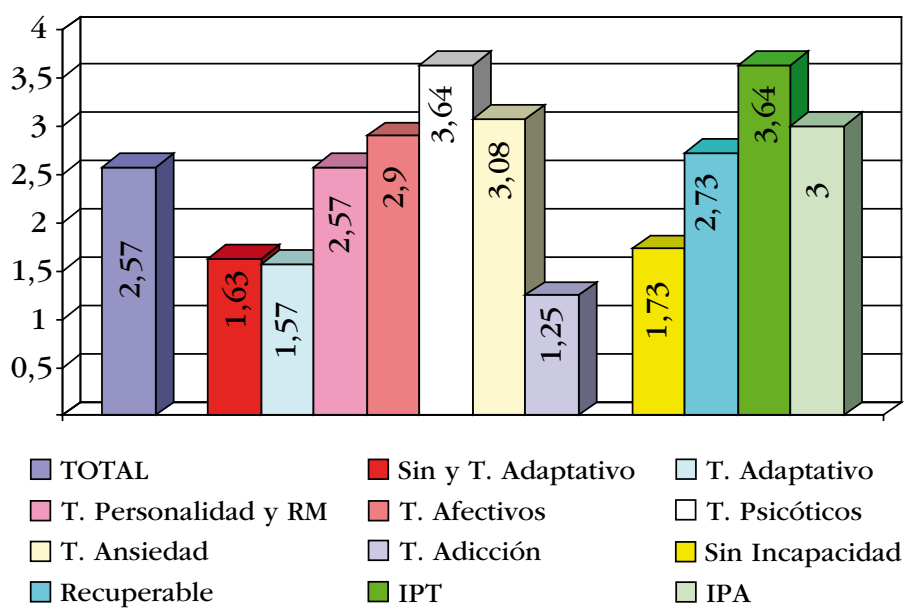

A los pacientes con sintomatología psicótica se les aplicó la PANSS ${ }^{31}$ en los casos en los que se presentaba una esquizofrenia y la situación clínica y de entrevista así lo aconsejaba. En total sólo se implementó el cuestionario en 6 de los 97 pacientes incluidos, por lo que no se presentan resultados al tratarse de una muestra excesivamente pequeña y poco representativa.

Como consecuencia de la valoración, se realiza una propuesta de evaluación, con los resultados del gráfico 15, que incluye los porcentajes. Cuando se compara como bloque a los que se le propone IP, tanto total para su profesión (IPT) como absoluta para cualquier profesión (IPA) frente a los otros grupos, se encuentra que el factor asociado principal es la vivencia de apoyo social (gráfico 16), con una clara asociación estadística $\left(\mathrm{X}^{2}=8,667 ; \mathrm{p}=0,034\right)$.

Los que no parecen incapacitados psiquiátricamente tienen 1,24 años de seguimiento, los que se les propone prórroga 5,64 años y los que se les orienta a IP 9,88 años. Existe diferencia con significación estadística entre los orientados a IP y los demás ( $\mathrm{t}=2,243$; $\mathrm{p}=0,028)$ y entre los orientados a prórroga y los de IP $(\mathrm{t}=-2,357 ; \mathrm{p}=0,024)$. Los antecedentes 
familiares psiquiátricos se muestran de forma distinta en el grupo sin incapacidad psíquica frente a los demás, gráfico 17 , de forma significativa $\left(X^{2}=7,339 ; p=0,007\right)$.

Gráfico 15. Propuesta de evaluación

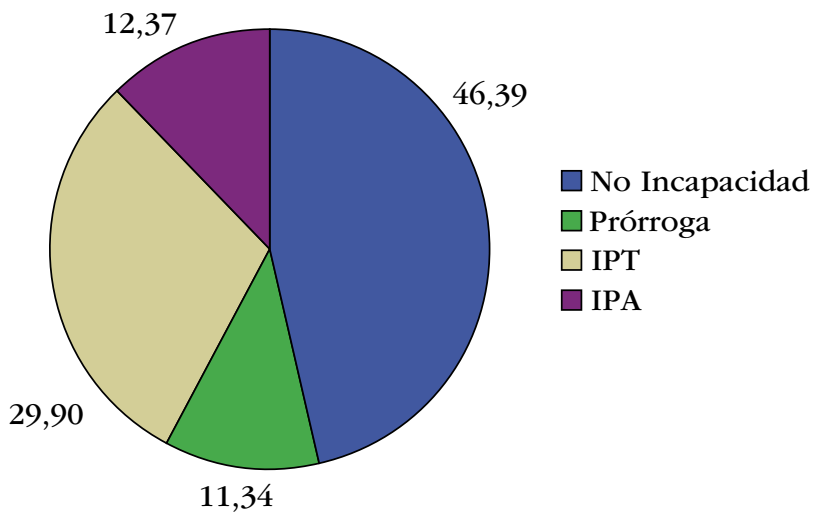

Gráfico 16. Apoyo social según propuestas

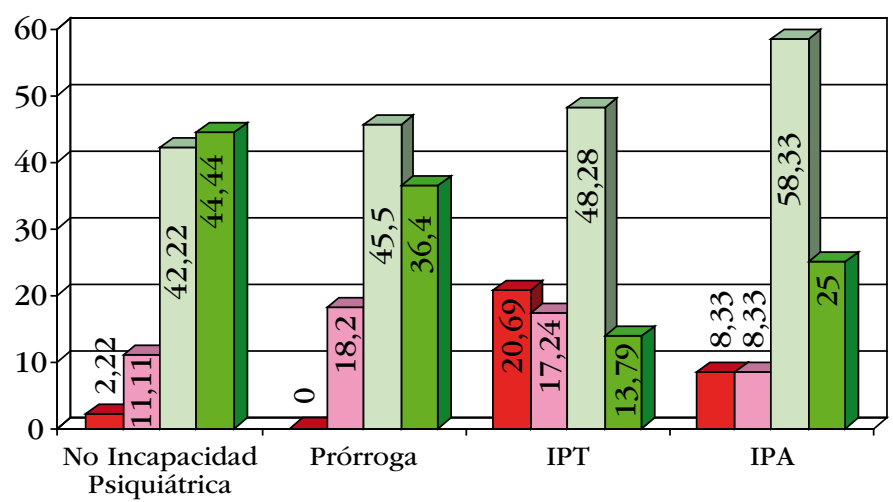

Nulo $\square$ Familia $\square$ Moderado $\square$ Positivo

Gráfico 17. Antecedentes Familiares según propuestas

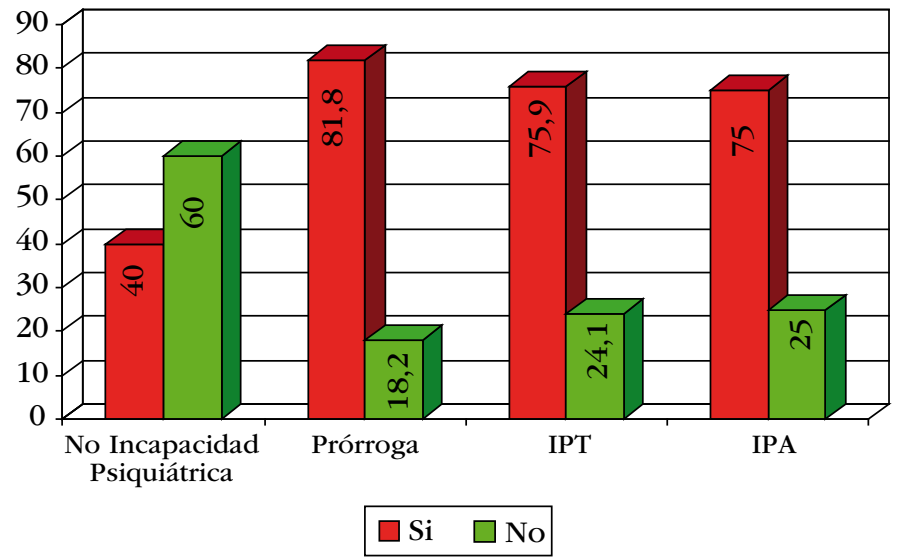

La distribución diagnóstica dentro de los grupos de propuesta (gráficos 18 a 21) varía en los distintos grupos de capacidad funcional propuesta. Entre los no incapacitados predomina el trastorno adaptativo. En la prórroga prevalece el trastorno afectivo, y los trastornos psicóticos aparecen directamente en las IP. No se pudo alcanzar significación estadística por el tamaño muestral ni corroborar datos respecto a la tendencia incapacitante de los trastornos de ansiedad como predictores de IP. 
Gráfico 18. Diagnósticos Grupales Principales en No Incapacidad Psiquiátrica

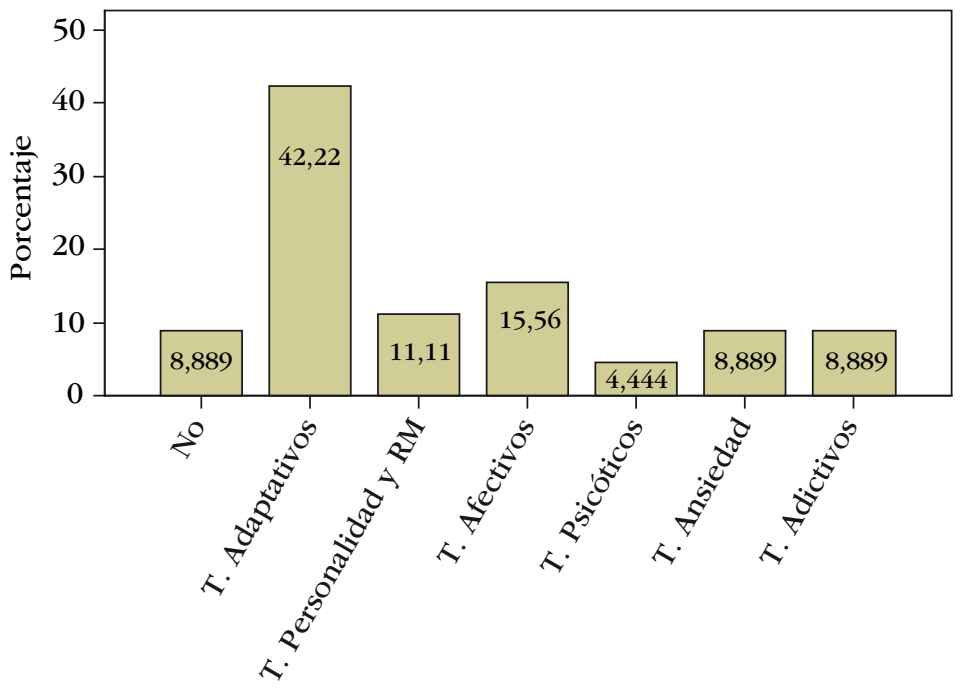

Gráfico 19. Diagnósticos Grupales Principales en Prórroga

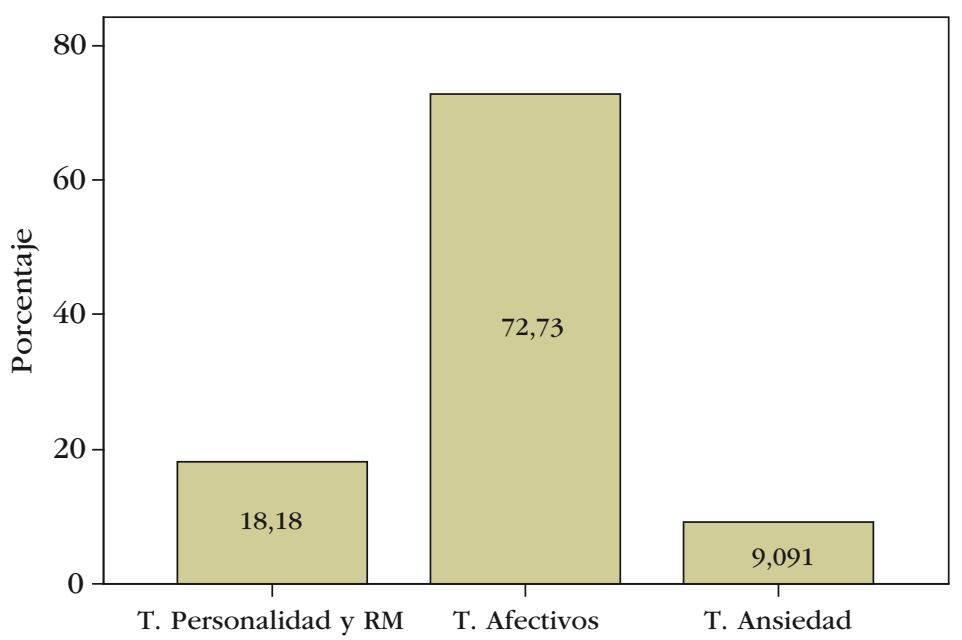

Gráfico 20. Diagnósticos Grupales Principales en IPT

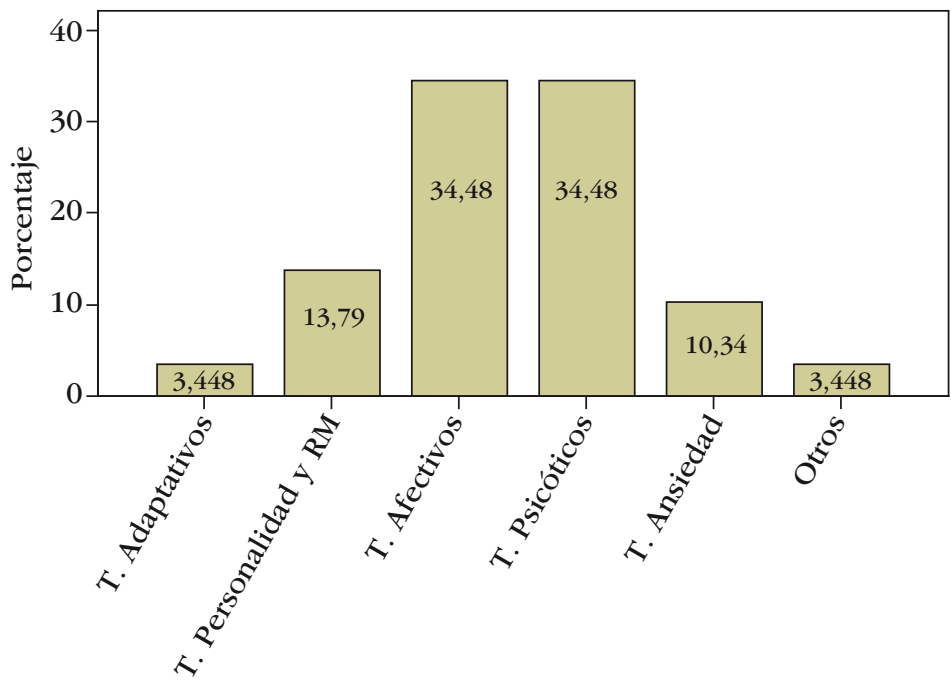


Gráfico 21. Diagnósticos Grupales Principales en IPA

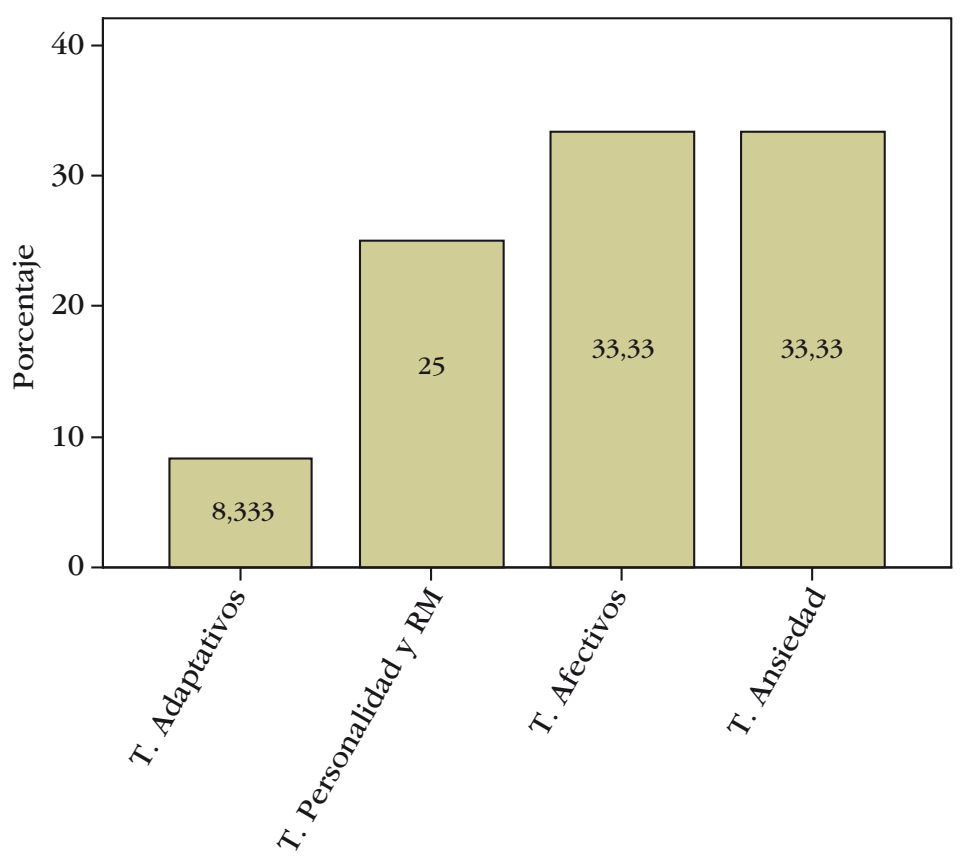

\section{DISCUSIÓN}

Las características sociodemográficas de la muestra concuerdan con lo apuntado en estudios previos, que otorgan mayor gasto y duración de la IT psiquiátrica a las mujeres y una concentración de edad en torno a los 35-55 años. ${ }^{19,33-34}$

Respecto al tipo de convivencia, resulta llamativa la diferencia significativa estadísticamente presentada en los trabajadores con patología psicótica (sin pareja) y los de patología depresiva (con pareja) frente al resto de pacientes. Así, los estudios que asocian vivir sin pareja con aumento del tiempo de IT, ${ }^{19}$ dato que no hemos encontrado, pueden estar basados en una muestra con predominancia psicótica.

Aunque algunos estudios consideran un factor protector tener hijos a cargo, ${ }^{35}$ no hemos encontrado concordancia al respecto, lo que impresiona de ser un factor de confusión posiblemente ligado al desempeño social.

El apoyo social sentido por los pacientes (gráfico 5), constituye un marcador de patología psiquiátrica grave que afecta especialmente a los grupos asociados a mayor deterioro social. Aunque se encuentran diferencias significativas por sexos, estas en realidad se deben a una mayor proporción de mujeres en el grupo adaptativo $(61,9 \%$ son mujeres frente al $38,1 \%$ de hombres).

Si bien los conflictos familiares no se asocian a ningún otro dato, los conflictos laborales parecen agruparse en una constelación formada por mujer, de mayor formación académica, con sintomatología afectiva moderada y diagnóstico de trastorno adaptativo. La proporción detectada de personas con conflicto laboral es similar a las detectadas en otros estudios al respecto. ${ }^{26}$

Sorprende que más de un tercio de la muestra alcanza la formación primaria como máximo, si bien es similar a lo detectado en otros estudios nacionales. ${ }^{16} \mathrm{Si}$ bien algunos autores han encontrado su asociación con cuadros depresivos, ${ }^{33}$ no es nuestro caso. La ausencia de asociaciones entre los grupos ocupacionales y el resto de variables es posible que se deba al tamaño muestral resultante al dividir la muestra global en subgrupos excesivamente pequeños y de baja representatividad. Por otro lado, comparada nuestra distribución con estudios similares que han utilizado los grupos principales de 
la CNO-94, ${ }^{26}$ se observa una gran similitud, excepto en las ocupaciones elementales no cualificadas. Cabe señalar que en dicho estudio la muestra provenía de trabajadores con sintomatología depresiva, que se ha señalado asociada a una percepción incrementada de exceso de demandas o saturación en otros estudios. ${ }^{36}$ Estudios en población norteamericana, ${ }^{25}$ en la que la prestación de la IT es muy distinta, señalan que los trastornos psiquiátricos disminuyen la productividad de todas las profesiones de forma similar, pero causan mayores periodos de interrupción de la actividad laboral en los profesionales, grupo probablemente equivalente a los profesionales científico y técnicos. Sin embargo, en población española se ha asociado la duración de la baja a trabajos semicualificados o no cualificados. ${ }^{19,27}$ Algunos otros trabajos, analizando los factores laborales como pronóstico de la reincorporación al trabajo ${ }^{37}$ consideran que, en realidad, lo importante no es el trabajo en sí sino cómo el trabajador se relaciona con su ambiente laboral cercano combinado con otros factores como la condición de salud en general.

Analizando los aspectos clínicos de la muestra, los antecedentes físicos parecen ser un indicador de severidad ${ }^{38}$ y de duración de la IT, ${ }^{27}$ y guarde relación con el aumento de la mortalidad del trastorno mental grave ${ }^{39}$ en todas las edades, géneros y razas. La asociación que presenta con la edad es lógica y coherente, dado que las enfermedades se van presentando y empeorando con el tiempo de vida. Resulta curioso que las enfermedades se asocien a mayor número de hijos, tenidos y a cargo, salvo que esta situación se encuentre de la misma forma correlacionada con la edad, aunque en nuestra muestra no sucede. Es importante destacar la asociación existente entre bajo nivel formativo y peor salud, como de antiguo viene siendo denunciado por la UNESCO y detectado a lo largo y ancho del planeta. ${ }^{39}$

El consumo de tabaco, primera causa de enfermedad prevenible en Occidente, resulta excesivo y preocupante, en torno a la mitad $(50,5 \%)$ de los pacientes estudiados fuma una cajetilla diaria $(18,84)$, a gran distancia del $34 \%$ de fumadores en la población española en esta franja de edad ${ }^{40} \mathrm{y}$ a todavía mayor distancia del $18 \%$ de la población neoyorquina. Este dato, junto a la elevada comorbilidad, permite entender la alta morbilidad presentada y la alta mortalidad de estos pacientes. ${ }^{41}$ La asociación del número de cigarrillos consumidos con el número de hijos, tenidos y a cargo, es un dato que no hemos encontrado en la bibliografía y para el que no encontramos explicación por el momento. El alcohol, segunda causa de enfermedad prevenible en Occidente, es un marcador de complejidad clínica, como muestra la asociación con el número de años en seguimiento psiquiátrico. Otras toxicomanías se presentan de forma similar en la población general y laboral. ${ }^{40,42}$

Los antecedentes familiares psiquiátricos son otro indicador de gravedad en la muestra explorada y se asocia con otras variables también marcadoras de gravedad como son los años en tratamiento psiquiátrico y el número de diagnósticos psiquiátricos, además de las escalas psicométricas empleadas. Estos datos en conjunto son coherentes y no han sido señalados en la bibliografía disponible por el momento.

La valoración de los diagnósticos referidos en la derivación del INSS y los realizados con criterios DSM-IV-TR tras la evaluación psiquiátrica muestran bastante concordancia. Otros estudios han señalado concordancias diagnósticas más bajas, $65 \%$, entre médicos de contingencia común de una mutua en Málaga y un psiquiatra consultor externo. ${ }^{43,44}$ Este dato de la discrepancia diagnóstica se ve acrecentado en algunas zonas con la mala coordinación entre Salud Mental y Atención Primaria, como sucede en Murcia y Alicante. ${ }^{12}$ Incluso la discrepancia diagnóstica está descrita dentro del propio médico según rellene el parte de baja o elabore un informe, ${ }^{45}$ coincidiendo sólo en el $92 \%$. Incluso dentro de los propios psiquiatras, existen notables diferencias en función de que se dediquen a la clínica o a la forensía, ${ }^{46}$ estos últimos con formación específica en evaluación de la discapacidad. Es por esto por lo que se ha propuesto incluir la formación en evaluación de la capacidad funcional en los programas de los futuros psiquiatras y en la formación médica continuada. ${ }^{47}$ 
Las diferencias porcentuales entre los grupos diagnósticos del INSS y el especializado, provienen fundamentalmente de la reasignación de procesos que son derivados sin información respecto a ninguna patología, de forma global hacia todas las demás patologías en más de $2 / 3$ de los casos. Asimismo se produce una migración de casos desde los diagnósticos de trastornos afectivos y trastornos de ansiedad hacia los trastornos adaptativos, dado que es fácil confundir el síntoma con la enfermedad. De esta forma, el grupo diagnóstico principal lo constituyen los trastornos afectivos (depresiones y trastorno bipolar) con un $29,9 \%$, y el segundo los trastornos adaptativos con un $21,6 \%$. Estudios con pacientes similares ${ }^{26}$ expresan distribuciones similares en algunos grupos, como los afectivos o psicóticos, pero se muestran confusión en el grupo diagnóstico ansiedad e infravaloran el eje II.

Los pacientes con trastorno adaptativo o sin patología identificada presentan un conjunto de características distintivas que no se habían señalado en estudios previos como el sentimiento de apoyo social, el número de codiagnósticos psiquiátricos y los años en seguimiento psiquiátrico, lo que confieren una carátula de levedad funcional. Sin embargo en algún estudio se ha ligado este diagnóstico con IT de larga duración cuando asocian comorbilidad o trabajos de "cuello azul". ${ }^{27}$

Los trastornos afectivos, que constituyen el grupo más frecuente en la IT de larga duración, se han asociado a una mayor tendencia a la incapacitación cuando se asocian otras patologías, físicas o psíquicas, ${ }^{48}$ de la misma forma que sucede con los trastornos de ansiedad. ${ }^{49}$ Estos dos grupos junto a los trastornos psicóticos confieren el grupo principal de la propuesta de incapacidad permanente, y suelen incluirse en las situaciones de Trastorno Mental Grave. ${ }^{39}$

La utilización de escalas psicométricas ofrece la oportunidad de cuantificar el deterioro y nivel de funcionamiento, conceptos conexos pero con amplias diferencias, que en algunos estudios ${ }^{50}$ se han utilizado para evaluar los factores asociados a la vuelta al trabajo tras baja psiquiátrica prolongada. Dentro del protocolo utilizado para la evaluación funcional y diagnóstica de estos pacientes, se aplican distintos cuestionarios orientados al funcionamiento, a la discapacidad y al diagnóstico. Así, orientado hacia la actividad global, aunque se le achaca habitualmente estar contaminado por la sintomatología clínica, utilizamos la EEAG, eje V de la DSM-IV-TR, ${ }^{29}$ para adquirir una primera visión global de la capacidad de funcionamiento. Se ha mostrado en nuestro estudio capaz de separar a los pacientes con cuadros adaptativos o sin patología de los demás grupos. La escala de depresión de Montgomery Asberg (MADRS), ${ }^{31}$ es heteroaplicada, lo que dificulta la exageración u ocultación de sintomatología y nos permite identificar a los pacientes depresivos. Estas dos escalas nos permiten separar claramente a los pacientes con limitación funcional de los que no la tienen.

La escala de discapacidad de la OMS, WHO-DAS, es una evaluación compleja y prolongada en su forma completa, si bien en el medio español existe una simplificación de Bobes ${ }^{31}$ que facilita su implementación y viene a comportarse como una escala de Likert de 6 grados entre el funcionamiento deseable, puntuación 0 , y 5 como grado máximo de afectación en cada una de las 4 subescalas en que se divide, cuidado personal (WODAS P), funcionamiento ocupacional (WODAS O), funcionamiento familiar (WODAS F) y funcionamiento social en un amplio sentido (WHODAS S). Dado el medio en el que nos encontramos y para potenciar los aspectos ocupacionales habíamos elegido la subescala ocupacional, WHODAS O. Esta escala tiene un comportamiento muy similar a la EEAG, distinguiendo los adaptativos de los demás y a los que no se les aprecia limitación del resto.

La valoración conjunta de los test aplicados, permite tomar una idea respecto a la posible gravedad, dadas las intensas correlaciones estadísticamente significativas entre las distintas escalas (tabla 5). De esta forma, se pueden considerar los resultados de los cuestionarios seleccionados como signos de gravedad clínica y de disfunción laboral.

La propuesta de evaluación realizada en forma de comentario final muestra influencia y asociación a determinados factores que no conocíamos previamente en la 
bibliografía. La vivencia de apoyo social (gráfico 16), el tiempo en seguimiento psiquiátrico, que correlaciona con las escalas evaluativas, y los antecedentes familiares psiquiátricos (gráfico 17) se comportan como marcadores de gravedad y de incapacidad, permitiéndonos escalonar las decisiones o facilitar la separación de los grupos.

Cada una de las propuestas realizadas tiene una patología principal distinta, indicando una diferente etiopatogenia y un curso evolutivo propio. El trastorno adaptativo que predomina en los no incapacitados se diferencia evolutivamente de los trastornos afectivos, que pueden requerir mayor tiempo de tratamiento, sin llegar a ser tan incapacitantes como los trastornos psicóticos. Los trastornos de ansiedad parecen ser poco limitantes, pero cuando lo son, lo hacen con gran intensidad, aunque no disponemos del tamaño muestral que nos permita afirmarlo con significación estadística.

Pese a que estudios previos asocian la incapacidad con la edad, de forma global ${ }^{18,53-54}$ o al superar los 50 años ${ }^{55}$ o el género masculino, ${ }^{53-54}$ factores económicos como la base reguladora, el nivel formativo alcanzado o el puesto de trabajo, ${ }^{54,56}$ no se ha conseguido detectar ninguna de estas asociaciones en la muestra estudiada.

\section{CONCLUSIONES}

El 46,39\% de los pacientes evaluados no presenta criterios de incapacidad funcional psiquiátrica. Este grupo capacitado suele estar compuesto por personas con trastornos adaptativos, buen apoyo social, poco tiempo en seguimiento psiquiátrico y no contar con antecedentes familiares psiquiátricos.

Los diagnósticos previos y posteriores a la evaluación psiquiátrica presentan una concordancia aceptable. En el trastorno adaptativo suele haber menos codiagnósticos psiquiátricos, menos tiempo en tratamiento psiquiátrico, puntúa con menor intensidad en las escalas, y se suele asociar al grupo sin indicación psiquiátrica de incapacidad y al conflicto laboral. Los trastornos afectivos son el grupo más frecuente y se suelen representar en las propuestas de prórroga y de IP.

Las escalas psicométricas permiten diferenciar al trastorno adaptativo de los demás (EEAG y WHODAS O) y a los casos sin incapacidad psíquica. MADRS señala los cuadros afectivos, distinguiéndolos de los demás, incluidos ansiosos. Estas escalas correlacionan entre sí.

Se han detectado marcadores de gravedad y de disfunción laboral y social. El apoyo social referido por el paciente es un marcador de patología psiquiátrica y de disfunción laboral, que distingue a la IP del resto. La ausencia de pareja en el grupo psicótico es un dato de disfunción social.

Los antecedentes familiares psiquiátricos son un marcador de gravedad, que se asocia al tiempo de seguimiento psiquiátrico, número de diagnósticos psiquiátricos y puntuación en las escalas psicométricas.

El tiempo de seguimiento psiquiátrico, además, es un marcador de cronicidad y disfunción social evidenciable a través de un bajo EEAG. Permite escalonar la IP de la prórroga y de la capacidad laboral psiquiátrica.

Clínicamente se detecta una alta comorbilidad expresada, que se asocia con la edad y con la baja formación académica. Los pacientes evaluados presentan un porcentaje de tabaquismo grave en todos sus grupos, signo de la ausencia de políticas de prevención sanitaria sobre este colectivo. Los pacientes con alcoholismo presentan un aumento de la complejidad diagnóstica y terapéutica asociado a una mayor cronicidad expresada en el tiempo de seguimiento psiquiátrico.

Si bien hemos obtenido nuevos datos y confirmado algunos, aún existen distintos campos por estudiar en este terreno, en especial en el área económica y las resoluciones 
definitivas de la capacidad laboral, por lo que es preciso continuar con estudios similares que puedan confirmar, perfilar o añadir nuevos datos a los aquí señalados.

\section{REFERENCIAS BIBLIOGRÁFICAS}

1. Wittchen, HU; Jacobi, F; Rehm, J; Gustavsson, A; Svensson, M; Jönson, B; Olesen, J; Allgulander, C; Alonso, J; Faravelli, C; et al. The size and burden of mental disorders and other disorders of the brain in Europe 2010. European Neuropsychopharmacology (2011) 21, 655-679.

2. Knudsen, AK; Overland, S; Hotopf, M; Mykletum, A. Lost working years due to mental disorders: An analysis of the Norwegian disability pension registry. Plos One, www.plosone.org August 2012, Vol 7, Issue 8 , e42567.

3. Leo, RJ; Del Regno, P. Social Security claims of psychiatric disability: elements of case adjudication and the role of primary care physicians. Primary Care Companion Journal of Clinical Psychiatry 2001; 3 (6):255-262.

4. Gustavsson, A; Svensson, M; Jacobi, F; Allgulander, C; Alonso, J; Beghi, E; Dodel, R; et al. Cost of disorders of the brain in Europe 2010. European Neuropsychopharmacology (2011) 21, 718-779.

5. The London School of Economics and Political Science. The depression report: A new deal for depression and anxiety disorders. LSE Research Online; 2006: http://cep.lse.ac.uk/textonly/research/mentalhealth/ DEPRESSION_REPORT_LAYARD.pdf

6. Rovira, J; Albarracin, G; Salvador-Carulla, L; Rejas, J; Sánchez-Iriso, E; Cabasés, JM. The cost of Generalized Anxiety disorder in primary care settings: Results of the ANCORA study. Community Mental Health Journal; DOI 10.1007/s10597-012-9503-4 (apr-2012).

7. Salvador-Carulla, L; Bendeck, M; Fernández, A; Alberti, C; Sabes-Figuera, R; Molina, C; Knapp, M. Cost of depression in Catalonia (Spain). Journal of Affective Disorders (2011), doi: 10.1016/j.jad.2011.02.019.

8. Sicras-Mainar, A; Blanca-Tamayo, M; Gutiérrez-Nicuesa, L; Salvatella-Pasant, J; Navarro-Artieda, R. Impacto de la morbilidad, uso de recursos y costes en el mantenimiento de la remisión de la depresión mayor en España: estudio longitudinal de ámbito poblacional. Gaceta Sanitaria 2010; 24 (1):13-19.

9. Pamias Massana, M; Crespo Palomo, C; Gisbert Gelonch, R; Palao Vidal, DJ. El coste social de la depresión en la ciudad de Sabadell (2007-2008). Gaceta Sanitaria 2012; 26 (2):153-158.

10. Mykletun, A; Overland, S; Dahl, AA; et al. A population-based cohort study of the effect of common mental disorders on disability pension awards. American Journal of Psychiatry aug-2006; 163:1412-1418.

11. Cemalovic, N. Evaluation of working capacity in case of mental disoders. MED ARH 2011; 65 (5): 304-305.

12. Araña Suárez, SM. Diagnósticos y prescripciones en la salud mental, atención primaria e incapacidad laboral temporal. Realizado en 2008. Disponible en: http://www.seg-social.es/prdi00/groups/public/ documents/binario/116335.pdf.

13. Delclós, J; García, S; López, JC; et al. Duración de la incapacidad temporal por contingencia común por grupos diagnósticos. Archivos de Prevención de Riesgos Laborales 2010; 13 (4):180-187.

14. Vilariño Rodríguez, DM; Vicaria Arroyo, FJ. Trastorno de ansiedad-depresión en contingencia común en la ciudad de Málaga. Disponible en http://diposit.ub.edu/dspace/bitstream/2445/13327/1/ TRASTORNOS\%20ANSIEDAD-DEPRESION.\%20MME.\%20word.pdf.

15. Jornadas de Incapacidad Temporal de la Sociedad Española de Medicina General, Madrid 2009. Disponible en http://www.semg.es/doc/comunidades/madrid/it_ano_cambio.pdf.

16. 16. Rivas Recio, JR; Sesé Madrazo, C. Estudio sobre la incapacidad laboral permanente: Valencia 20022005. Medicina y Seguridad en el Trabajo 2006; Vol LII, $n^{\circ}$ 204:7-42.

17. Estadísticas sobre IP (ejercicio 2011). Seguridad Social: http://www.seg-social.es/Internet_1/Estadistica/ Est/Otras_Prestaciones_de_la_Seguridad_Social/Incapacidad_Permanente/Incapacidad_Permanente/ index.htm.

18. Blank, L; Peters, J; Pickvance, S; Wilford, J; MacDonald, E. A systematic review of the factors wich predict return to work for people suffering episodes of poor mental health. Journal of Occupational Rehabilitation (2008) 18:27-34.

19. Vaquero Abellán, M. Factores que influyen en la incapacidad temporal de larga duración: propuesta de screening y de intervención. Disponible en http://www.seg-social.es/prdi00/groups/public/documents/ binario/168544.pdf.

20. Lexis, MAS; Jansen, NWH, van Amelsvoort, LGPM; et al. Prediction of long-term sickness absence among employees with depressive complaints. Journal of Occupational Rehabilitation (2012) 22:262-269. 
21. Sogaard, HJ; Bech, P. Psychometric analysis of common mental disorders - Screening Questionnaire (CMD-SQ) in long-term sickness absence. Sacandinavian Journal of Public Health 2009 Nov; 37 (8):858-863.

22. Calvo Bonacho, E. Duración de la incapacidad temporal asociada a diferentes patologías en trabajadores españoles: discusión acerca de la ITCC en los trastornos mentales. Madrid 2010. Disponible en http:// www.seg-social.es/prdi00/groups/public/documents/binario/146666.pdf.

23. Pastrana Jiménez, JI. Aspectos psiquiátricos y sociales del mobbing. Revista del Centro de Estudios Jurídicos, Ministerio del Interior - Universidad Complutense de Madrid, Septiembre de 2004.

24. Clasificación nacional de Ocupaciones, versión 2011: CNO-2011. Disponible en http://www.ine.es/daco/ daco42/clasificaciones/cno11_notas.pdf.

25. Kessler, R; Frank, R. The impact of psychiatric disorders on work loss days. Psychological Medicine, 1997, 27, 861-873.

26. Rodríguez Ortiz de Salazar, B; Gómez Gálligo, A; García Parra, A; López Gómez, I. Sintomatología depresiva asociada al trabajo como causa de incapacidad temporal en la Comunidad de Madrid. Mapfre Medicina, 2005, 16, 3: 184-194.

27. Catalina Romero, C; Pastrana Jiménez, JI; Tenas López, MJ; et al. Long-term sickness absence due to adjustment disorder. Occupational Medicine doi:10.1009/occmed/kqs043.

28. Haukenes, I; Mykletun, A; Knudsen, AK; Hansen, HT; Maeland, JG. Disability pension by occupational class - the impact of work-related factors: The Hordaland Health Study Control. BMC Public Health 2011, 11:406.

29. American Psychiatric Association. DSM-IV-TR. Editorial Masson, Barcelona 2002.

30. Clasificación Internacional de Enfermedades, $9^{a}$ versión, Modificación Clínica: CIE-9-MC. Disponible en http://www.msc.es/estadEstudios/estadisticas/docs/Manual_de_codificacion.pdf.

31. Bobes, J; González, MP; Sáinz, PA; et al. Instrumentos básicos para la práctica de la psiquiatría clínica. Grafo SA, Bilbao 2000

32. Asociación Médica Mundial. Declaración de Helsinki, versión 2010. Disponible en http://www.wma.net/ es/30publications/10policies/b3/

33. Veronese, A; Ayuso-Mateos, JL; Cabello, M Chaterji, S; Nuevo, R. Work disability and depressive disorders: impact on the European population. American Journal of Physician and Medical Rehabilitation, 2012 feb, 91 (13 Suppl 1): S62-8.

34. Molina, JM; Fernández, M; Zúñiga, A. Perfil clínico y sociodemográfico de la incapacidad temporal de larga evolución de causa mental: estudio transversal. medicina del Trabajo 19, 2; julio 2010 (21-32).

35. Sogaard, HJ, Bech, P. Psychiatric disorders in long-term sickness absence - a population based crosssectional study. Scandinavian Journal of Public Health, 2009 Sep, 37 (7):682-689.

36. Kivimaki, M; Vahtera, J; Kawachi, I et al. Psychosocial work environment as a risk factor for absence with a psychiatric diagnosis: An instrumental-variables analysis. American Journal of Epidemiology2010; 172: $167-172$

37. Nielsen, MB; Baltmann, U; Madsen, IE; et al. Health, work, and personal-related predictors of time to return to work among employees with mental health problems. Disability Rehabilitation 2012; 34 (15): 1311-1316.

38. Kessler, RC; Chiu, WT; Demler, O; Walters, EE. Prevalence, severity, and comorbidity of 12-month DSM-IV disorders in the National Comorbidity Survey Replication. Archives of General Psychiatry, 2005;62:617-627.

39. Lewin, K; Little, A; Colclough, C. Los efectos de la educación sobre los objetivos del desarrollo. Perspectivas 1983, vol XXIII, $n^{\circ}$ 4: 433-447.

40. INE. Anuario estadístico de España 2012. Disponible en: http://www.ine.es/prodyser/pubweb/anuario12/ anu12_05salud.pdf.

41. Chang, CK; Hayes, RD; Breadbent, M; et al. All-cause mortality among people with serious mental illness (SMI), substance use disorders, and depressive disorders in southeast London: a cohort study. BMC Psychiatry 2010, 10:77.

42. Navarro Botella, J; Gómez González, E. La incidencia de las drogas en el mundo laboral en la Comunidad de Madrid. Cauce editorial, Madrid 1998.

43. Almarcha Vicente, MI; Aparicio Navarro, JV; Enguix Monzó, V. Influencia del peritaje en la duración de la IT en la patología psiquiátrica más frecuente en contingencias comunes. Master de medicina evaluador 2009. Disponible en: http://diposit.ub.edu/dspace/bitstream/2445/13328/1/TRASTORNOS\%20 ADAPTATIVOS.PERITAJE.MME.word.pdf.

44. González Klett, M; Mata Hernández, I; Mena García, E. Trastornos de ansiedad-depresión en el entorno de la contingencia común. Master de medicina evaluadora 2009. Disponible en: http://diposit.ub.edu/ dspace/bitstream/2445/13327/1/TRASTORNOS\%20ANSIEDAD-DEPRESION.\%20MME.\%20word.pdf. 
45. Castejón, J; Benavides, FG; Gimeno; Company, A; Fábrega, O; Funes, J. Calidad del diagnóstico médico en la certificación de la incapacidad temporal por enfermedad común y accidente no laboral. Atención Primaria 2006; 373(3): 142-147.

46. Christopher, PP; Arikan, R; Pinals, DA; et al. Evaluating psychiatric disability: Differences by forensic expertise. Journal of the American Academy of Psychiatry \& Law 2011; 39:183-188.

47. Gold, LH. Commentary: Challenges in providing psychiatry disability evaluations. Journal of the American Academy of Psychiatry \& Law 2011; 39:189-193.

48. Ahola, K; Virtanen, M; Honkonen, T; et al. Common mental disorders and subsequent work disability: A population-based Health 2000 Study. Journal of Affective Disorders 134 (2011)365-372.

49. Catalina Romero, C; Martínez Muñoz, P; Quevedo Aguado, L; et al. Predictores de la duración de la incapacidad temporal por contingencias comunes en los trastornos de ansiedad. Gaceta Sanitaria 2012, doi:10.1016/j.gaceta.2011.12.008.

50. Mujizer, A; Brouwer, S; Geertzen, JH; Groothoff, JW. Exploring factors relevant in the assessment of the return-to-work process of employees on long-term sickness absence due to a depressive disorder; a focus group study. BMC Public Health 2012, 12:103.

51. Virtanen M, Kawachi, I; Oksanen, T; et al. Socio-economic differences in long-term psychiatrich work disability: prospective cohort study of onset, recovery and recurrence. Occupational and Environmental Medicine 2011 Nov, 68 (11):791-798.

52. Mykletun, A; Overland, S; Dahl, AA; et al. A population-based cohort study of the effect of common mental disorders on disability pension awards. American journal of Psychiatry 2006; 163:1412-1418.

53. Bratberg E; Gjesdal, S; Maeland, JG. sickness absence with psychiatric diagnoses. individual and contextual predictors of permanent disability. Health Place 2009 Mar, 15 (1):308-314.

54. Cornelius, LR; van der Klink, JJL; Groothoff, JW. Prognostic factors of long term disability due to mental disorders: A systematic review. Journal of Occupational Rehabilitation (2011) 21:259-274.

55. Nieuwenhuijsen, K;Verbeek, JHAM; de Boer, AGEM; et al. Predicting the duration of sickness absence for patients with common mental disorders in occupational health care. Scandinavian journal of Work and Environmental Health 2006, 32 (1): 67-74.

56. Gjesdal S; Ringdal, R; Haug, K; et al. Predictors of disability pension in long-term sickness absence. European Journal of Public Health 2004; 14:398-405.

\section{AGRADECIMIENTOS}

Quisiéramos mostrar nuestra gratitud hacia Luis Quevedo, cuya ayuda en la localización de la documentación y bibliografía escondida fue fundamental. 